ANNALES

POLONICI MATHEMATICI

LXXIX.1 (2002)

\title{
Solutions to some nonlinear PDE's in the form of Laplace type integrals
}

\author{
by MARIA E. Pliś (Kraków)
}

\begin{abstract}
A nonlinear equation $P(D) u=\alpha u^{m}$ in 2 variables is considered. A formal solution as a series of Laplace integrals is constructed. It is shown that assuming some properties of Char $P$, one gets the Gevrey class of such solutions. In some cases convergence "at infinity" is proved.
\end{abstract}

1. Introduction. In $[\mathrm{P}-\mathrm{Z}]$ we have considered a nonlinear equation

$$
P(D) u=\sum_{j=1}^{\infty} c_{j} u^{j}
$$

with all $c_{j}$ constant (complex or real), $D=\left(\partial / \partial x_{1}, \partial / \partial x_{2}\right)$, and we have found a formal solution of (1) represented at infinity as a formal sum of Laplace type integrals. In general, solutions of this type are divergent. In this work we show that in some cases these formal solutions are in some formal Gevrey class. Similar problems for some nonlinear singular partial differential equations were studied in [G-T].

This paper is a continuation of [P-Z], but we recall all definitions and notations. We restrict our attention to the case

$$
P(D) u=\alpha u^{m}
$$

for some fixed $m \in \mathbb{N}, m \geq 2$, and $\alpha$ a real or complex number, where $P$ is a polynomial of two variables. In what follows, for $a=\left(a_{1}, a_{2}\right)$ and $b=\left(b_{1}, b_{2}\right)$, we write $a \leq b($ resp. $a<b)$ whenever $a_{i} \leq b_{i}\left(\right.$ resp. $\left.a_{i}<b_{i}\right)$ for $i=1,2$ and $a b$ is the scalar product. For $\eta \in \mathbb{R}$ we write $\eta a$ instead of $\eta a_{1}+\eta a_{2}$.

The assumptions on the left-hand side of (2) are given below. Set Char $P$ $=\left\{z \in \mathbb{C}^{2}: P(z)=0\right\}$. We assume that there exists an unbounded curve $Z \subset$ Char $P \cap \mathbb{R}_{+}^{2}$ such that:

(i) $2 Z \cap Z=\emptyset$;

2000 Mathematics Subject Classification: 35C10, 35C15, 44A10, 46F12.

Key words and phrases: Laplace distributions, convolution equations, Gevrey classes. 
(ii) $k Z \subset n Z$ for $2 \leq n<k ; n, k \in \mathbb{N}$ (here $k Z=Z+\ldots+Z$ is the algebraic sum of $k$ summands);

(iii) $|P(z)| \geq 1$ for $z \in 2 Z$;

(iv) for every $\eta>0$ there exists a constant $M_{\eta}$ such that for every $c=\left(c_{1}, c_{2}\right)$ with $c_{i} \geq \eta, \int_{Z} e^{-c x} d x \leq M_{\eta}$.

The integral in (iv) is a line integral over $Z$.

Following [S-Z], for $a \in \mathbb{R}^{2}$ we define

$$
L_{a}=\left\{\phi \in C^{\infty}\left(\left(\overline{\mathbb{R}}_{+}\right)^{2}\right): \sup _{x \in\left(\overline{\mathbb{R}}_{+}\right)^{2}}\left|e^{-a x}(\partial / \partial x)^{\nu} \phi(x)\right|<\infty, \nu \in \mathbb{N}_{0}^{2}\right\}
$$

with convergence defined by the seminorms

$$
\|\phi\|_{a, \nu}=\sup _{x \in\left(\overline{\mathbb{R}}_{+}\right)^{2}}\left|e^{-a x}(\partial / \partial x)^{\nu} \phi(x)\right|,
$$

and for $\omega \in \mathbb{R}^{2}$ we define

$$
L_{(\omega)}=\underset{a<\omega}{\lim _{a}} L_{a}
$$

equipped with the inductive limit topology. The dual space $L_{(\omega)}^{\prime}$ is called the space of Laplace distributions on $\left(\overline{\mathbb{R}}_{+}\right)^{2}$.

2. Construction of solutions. The function $e^{-x z}$ as a function of $z$ ( $x$ fixed) belongs to the space $L_{a}$ for every $a \geq-x$, so it belongs to $L_{(\omega)}$ for every $\omega>-x$. We look for a solution of (2) in the form

$$
u(x)=T\left[e^{-x z}\right]
$$

for some Laplace distribution $T$. Applying $P(D)$ to $u$ of the form (3) we arrive at the convolution equation

$$
P(z) T=\alpha T^{* m} .
$$

Here $T^{* m}$ stands for $T * \ldots * T$ with $m$ factors.

We modify slightly this equation by multiplying the right-hand side by a factor $\varepsilon \in(0,1]$, so we consider

$$
P(z) T=\alpha \varepsilon T^{* m},
$$

and we look for $T$ in the form of a formal series

$$
T=\sum_{k=0}^{\infty} \varepsilon^{k} T_{k}
$$

of Laplace distributions $T_{k}$. 
Inserting (6) in (5) we obtain

$$
\begin{aligned}
\sum_{k=0}^{\infty} P(z) T_{k} \varepsilon^{k} & =\varepsilon \alpha \sum_{k=0}^{\infty} \varepsilon^{k} \sum_{k_{1}+\ldots+k_{m}=k} T_{k_{1}} * \ldots * T_{k_{m}} \\
& =\sum_{k=1}^{\infty} \varepsilon^{k} \alpha \sum_{k_{1}+\ldots+k_{m}=k-1} T_{k_{1}} * \ldots * T_{k_{m}},
\end{aligned}
$$

therefore, we get the recurrence system

$$
\begin{aligned}
& P(z) T_{0}=0, \\
& P(z) T_{k}=\alpha \sum_{k_{1}+\ldots+k_{m}=k-1} T_{k_{1}} * \ldots * T_{k_{m}} \quad \text { for } k \geq 1 .
\end{aligned}
$$

Fix $\eta>0, b=\left(b_{1}, b_{2}\right) \in \mathbb{R}^{2}$ and set $\omega=-\eta-b=\left(-\eta-b_{1},-\eta-b_{2}\right)$. Let $M_{\eta}$ be the constant in (iv) and $\Phi$ a function in $C^{\infty}\left(\mathbb{R}_{+}^{2}\right)$ satisfying

$$
0<\Phi(x) \leq C e^{b x} \quad \text { for all } x \in \mathbb{R}_{+}^{2},
$$

for some constant $C<|\alpha|^{-1 /(m-1)} M_{\eta}^{-1}$.

Exactly as in $[\mathrm{P}-\mathrm{Z}]$ we define a Laplace distribution $T_{0}$ by the formula

$$
T_{0}[\phi]=\int_{Z} \phi(x) \Phi(x) d x
$$

for every test function $\phi \in L_{(\omega)}$.

One can see immediately that $T_{0}$ is a solution of $(7), T_{0} \in L_{(\omega)}^{\prime}$ and $\operatorname{supp} T_{0} \subset Z$.

Definition (10) and properties of convolution imply that for $p \in \mathbb{N}$ and $\phi \in L_{a}$ with $a<\omega$,

$$
\left|T_{0}^{* p}[\phi]\right| \leq\left(C M_{\eta}\right)^{p} \sup _{z \in p Z}\left|\phi(z) e^{-a z}\right|
$$

Indeed,

$$
\begin{aligned}
T_{0}^{* p}[\phi] & =\int_{Z \times \ldots \times Z} \phi\left(z_{1}+\ldots+z_{p}\right) \Phi\left(z_{1}\right) \ldots \Phi\left(z_{p}\right) d z_{1} \ldots d z_{p} \\
& =\int_{Z^{p}} \phi\left(z_{1}+\ldots+z_{p}\right) e^{-a\left(z_{1}+\ldots+z_{p}\right)} \Phi\left(z_{1}\right) e^{a z_{1}} \ldots \Phi\left(z_{p}\right) e^{a z_{p}} d z_{1} \ldots d z_{p} .
\end{aligned}
$$

Hence,

$$
\left|T_{0}^{* p}[\phi]\right| \leq \sup _{z \in p Z}\left|\phi(z) e^{-a z}\right|\left(\int_{Z} \Phi(z) e^{a z} d z\right)^{p},
$$

which is due to the fact that $z_{1}+\ldots+z_{p} \in p Z$ for $z_{i} \in Z$. This gives (11) because $\int_{Z} \Phi(z) e^{a z} d z \leq C \int_{Z} e^{(a+b) z} d z \leq C M_{\eta}$, since $a+b<-\eta$.

LEMMA 1. Let $\Theta$ be a function in $C^{\infty}\left(\mathbb{R}_{+}^{2 k}\right), k \geq 2$, satisfying

$$
0<\Theta(z) \leq H e^{b\left(z_{1}+\ldots+z_{k}\right)}
$$


for some constants $H>0, b=\left(b_{1}, b_{2}\right) \in \mathbb{R}^{2}$, and for all $z=\left(z_{1}, \ldots, z_{k}\right)$, $z_{i} \in \mathbb{R}_{+}^{2}(i=1, \ldots, k)$. Let $T \in L_{(\omega)}^{\prime}$ be a Laplace distribution for $\omega=-b-\eta$ with $\eta>0$, given by

$$
T[\phi]=\int_{Z^{k}} \phi\left(z_{1}+\ldots+z_{k}\right) \Theta\left(z_{1}, \ldots, z_{k}\right) d z_{1} \ldots d z_{k}
$$

for $\phi \in L_{(\omega)}$. Then the Laplace distribution $S$ defined by

$$
S[\phi]=\int_{Z^{k}} \frac{\phi\left(z_{1}+\ldots+z_{k}\right)}{P\left(z_{1}+\ldots+z_{k}\right)} \Theta\left(z_{1}, \ldots, z_{k}\right) d z_{1} \ldots d z_{k},
$$

with $|P(z)| \geq 1$ for $z \in 2 Z$, solves the equation $P(z) S=T$, and for $\phi \in L_{a}$ with $a<\omega$,

$$
|S[\phi]| \leq \sup _{u \in k Z}\left|\phi(u) e^{-a u}\right| H M_{\eta}^{k} .
$$

Proof. It follows from assumption (iii) that formula (13) makes sense. If $\phi \in L_{(\omega)}$, then also $P \phi \in L_{(\omega)}$. Therefore

$$
(P S)[\phi]=S[P \phi]=\int_{Z^{k}} \phi\left(z_{1}+\ldots+z_{k}\right) \Theta\left(z_{1}, \ldots, z_{k}\right) d z_{1} \ldots d z_{k}=T[\phi],
$$

and for $\phi \in L_{a}$ with $a<\omega$ we obtain

$$
\begin{aligned}
|S[\phi]| \leq & \int_{Z^{k}}\left|\phi\left(z_{1}+\ldots+z_{k}\right)\right| e^{-a\left(z_{1}+\ldots+z_{k}\right)} \\
& \times\left|\Theta\left(z_{1}, \ldots, z_{k}\right)\right| e^{a\left(z_{1}+\ldots+z_{k}\right)} d z_{1} \ldots d z_{k} \\
\leq & \sup _{u \in k Z}\left|\phi(u) e^{-a u}\right| H \int_{Z^{k}} e^{(b+a)\left(z_{1}+\ldots+z_{k}\right)} d z_{1} \ldots d z_{k} \\
\leq & \sup _{u \in k Z}\left|\phi(u) e^{-a u}\right| H\left(\int_{Z} e^{(b+a) z} d z\right)^{k}
\end{aligned}
$$

and by (iv) we get (14), because $-(a+b)>\eta$.

If $T$ is as in Lemma 1, then we denote by $\frac{1}{P} T$ the distribution $S$ given by (13).

Lemma 2. Let $m \in \mathbb{N}, m \geq 2$ and $\left\{B_{k}\right\}_{k \in \mathbb{N}}$ be a sequence of real numbers defined by the recurrence formulas

$$
\begin{aligned}
& B_{1}=1, \\
& B_{k}=\sum_{r=1}^{\widetilde{m}}\left(\begin{array}{c}
m \\
r
\end{array}\right) \sum_{\substack{m_{1}+\ldots+m_{r}=k-1 \\
m_{i} \geq 1}} B_{m_{1}} \ldots B_{m_{r}} \quad \text { for } k>1,
\end{aligned}
$$

where $\widetilde{m}=\min \{m, k-1\}$. Then $B_{k} \leq((m-1) k) !$. 
Proof. The proof is by induction on $k$. Obviously, for every $m \geq 2$, $B_{1}=1 \leq(m-1)$ !. Suppose that $B_{s} \leq((m-1) s)$ ! for every $s<k$. From (15) it follows that

$$
\begin{aligned}
B_{k} & \leq \sum_{r=1}^{\widetilde{m}}\left(\begin{array}{c}
m \\
r
\end{array}\right) \sum_{\substack{m_{1}+\ldots+m_{r}=k-1 \\
m_{i} \geq 1}}\left((m-1) m_{1}\right) ! \ldots\left((m-1) m_{r}\right) ! \\
& \leq \sum_{r=1}^{\tilde{m}}\left(\begin{array}{c}
m \\
r
\end{array}\right)((m-1)(k-1)) !\left(\begin{array}{c}
k-2 \\
r-1
\end{array}\right) \\
& =((m-1)(k-1)) ! \sum_{r=1}^{\widetilde{m}}\left(\begin{array}{c}
m \\
r
\end{array}\right)\left(\begin{array}{c}
k-2 \\
r-1
\end{array}\right),
\end{aligned}
$$

due to the well known facts that the number of elements of $\left\{\left(m_{1}, \ldots, m_{r}\right)\right.$ : $\left.m_{1}+\ldots+m_{r}=n, m_{i} \geq 1, i=1, \ldots, r\right\}$ is $\left(\begin{array}{c}n-1 \\ r-1\end{array}\right)$, and that $p_{1} ! \ldots p_{r} ! \leq$ $\left(p_{1}+\ldots+p_{r}\right)$ !. It is sufficient to show that

$$
\begin{aligned}
\sum_{r=1}^{\tilde{m}}\left(\begin{array}{c}
m \\
r
\end{array}\right) & \left(\begin{array}{l}
k-2 \\
r-1
\end{array}\right) \leq \frac{((m-1) k) !}{((m-1)(k-1) !} \\
=((m-1) k-(m-2))((m-1) & k-(m-3)) \ldots((m-1) k) \\
& =\prod_{l=1}^{m-1}((m-1) k-(l-1)) .
\end{aligned}
$$

We check at once that

$$
\sum_{r=1}^{\tilde{m}}\left(\begin{array}{c}
m \\
r
\end{array}\right)\left(\begin{array}{l}
k-2 \\
r-1
\end{array}\right)=\left(\begin{array}{c}
k+m-2 \\
m-1
\end{array}\right)=\prod_{l=1}^{m-1} \frac{k+l-1}{l}
$$

and

$$
(m-1) k-(l-1)-\frac{k+l-1}{l}=\frac{[l(m-1)-1] k-\left(l^{2}-1\right)}{l} \geq 0
$$

for $l=1, \ldots, m-1$ and $k \geq 1$, and (16) is proved.

Let $\left(k_{1}, \ldots, k_{r}\right) \in \mathbb{N}_{0}^{r}$ be such that $k_{1}+\ldots+k_{r}=k-1, k \in \mathbb{N}, k_{i} \geq 1$ for $i=1, \ldots, r$. Then we define $k_{j}^{\prime}=(m-1)\left(k_{1}+\ldots+k_{j}\right)+j$ for $j=1, \ldots, r$, $k_{0}^{\prime}=0$ and $m_{k}=(m-1) k+1$. Clearly $k_{r}^{\prime}=m_{k}-(m-r)$. For $z=$ $\left(z_{1}, \ldots, z_{m_{k}}\right)$ we set $u_{k_{j}}=\left(z_{k_{j-1}^{\prime}+1}, \ldots, z_{k_{j}^{\prime}}\right)$ and $s\left(u_{k_{j}}\right)=z_{k_{j-1}^{\prime}+1}+\ldots+z_{k_{j}^{\prime}}$ for $j=1, \ldots, r$. Then we define $H_{k}: \mathbb{R}_{+}^{2 m_{k}} \rightarrow \mathbb{R}$ by the recurrence formula

$$
H_{1}\left(z_{1}, \ldots, z_{m}\right)=\frac{\Phi\left(z_{1}\right) \ldots \Phi\left(z_{m}\right)}{P\left(z_{1}+\ldots+z_{m}\right)},
$$


(18)

$$
\begin{aligned}
& H_{k}\left(z_{1}, \ldots, z_{m_{k}}\right) \\
= & \sum_{r=1}^{\widetilde{m}}\left(\begin{array}{c}
m \\
r
\end{array}\right) \sum_{\substack{k_{1}+\ldots+k_{r}=k-1 \\
k_{i} \geq 1}} \frac{H_{k_{1}}\left(u_{k_{1}}\right) \ldots H_{k_{r}}\left(u_{k_{r}}\right) \Phi\left(z_{k_{r}^{\prime}+1}\right) \ldots \Phi\left(z_{m_{k}}\right)}{P\left(s\left(u_{k_{1}}\right)+\ldots+s\left(u_{k_{r}}\right)+z_{k_{r}^{\prime}+1}+\ldots+z_{m_{k}}\right)} .
\end{aligned}
$$

LEMma 3. If $T_{0}$ is the Laplace distribution given by (10) then the distribution $T_{k}$ defined by

$$
T_{k}=\frac{\alpha}{P} \sum_{k_{1}+\ldots+k_{m}=k-1} T_{k_{1}} * \ldots * T_{k_{m}}
$$

for $k \geq 1$ satisfies the following conditions:

$$
\begin{gathered}
T_{k} \in L_{(\omega)}^{\prime} \quad \text { for } \omega=-b-\eta, \quad \text { and } T_{k} \text { solves (8); } \\
\operatorname{supp} T_{k} \subset m_{k} Z,
\end{gathered}
$$

where $m_{k}=(m-1) k+1 ;$ for every $\phi \in L_{a}$ with $a<\omega$,

$$
T_{k}[\phi]=\alpha^{k} \int_{Z^{m_{k}}} \phi\left(z_{1}+\ldots+z_{m_{k}}\right) H_{k}\left(z_{1}, \ldots, z_{m_{k}}\right) d z_{1} \ldots d z_{m_{k}}
$$

with $H_{k}$ given by (17) and (18);

$$
\int_{Z^{m_{k}}}\left|H_{k}\left(z_{1}, \ldots, z_{m_{k}}\right)\right| e^{a\left(z_{1}+\ldots+z_{m_{k}}\right)} d z_{1} \ldots d z_{m_{k}} \leq B_{k}\left(C M_{\eta}\right)^{m_{k}},
$$

and

$$
\left|T_{k}[\phi]\right| \leq|\alpha|^{k} B_{k}\left(C M_{\eta}\right)^{m_{k}} \sup _{u \in m_{k} Z}|\phi(u)| e^{-a u},
$$

where the sequence $B_{k}$ is given by (15).

Proof. The condition (20) follows immediately from the properties of convolution and from the assumptions on $P$.

In order to prove (21)-(24) we proceed by induction. For $k=1$ we have $T_{1}=\alpha(1 / P) T_{0}^{* m}$, so $\operatorname{supp} T_{1} \subset m Z$. By $(17)$,

$$
T_{1}[\phi]=\alpha \int_{Z^{m}} \phi\left(z_{1}+\ldots+z_{m}\right) H_{1}\left(z_{1}, \ldots, z_{m}\right) d z_{1} \ldots d z_{m},
$$

and

$$
\left|H_{1}\left(z_{1}, \ldots, z_{m}\right)\right| e^{a\left(z_{1}+\ldots+z_{m}\right)}=\frac{\Phi\left(z_{1}\right) e^{a z_{1}} \ldots \Phi\left(z_{m}\right) e^{a z_{m}}}{\left|P\left(z_{1}+\ldots+z_{m}\right)\right|} .
$$

Hence by (iii),

$$
\int_{Z^{m}}\left|H_{1}\left(z_{1}, \ldots, z_{m}\right)\right| e^{a\left(z_{1}+\ldots+z_{m}\right)} d z_{1} \ldots d z_{m} \leq\left(\int_{Z} \Phi(z) e^{a z} d z\right)^{m} .
$$

Therefore 
$\left|T_{1}[\phi]\right|$

$=|\alpha|\left|\int_{Z^{m}} \phi\left(z_{1}+\ldots+z_{m}\right) e^{-a\left(z_{1}+\ldots+z_{m}\right)} H_{1}\left(z_{1}, \ldots, z_{m}\right) e^{a\left(z_{1}+\ldots+z_{m}\right)} d z_{1} \ldots d z_{m}\right|$

$\leq|\alpha| \sup _{u \in m Z}|\phi(u)| e^{-a u}\left(C M_{\eta}\right)^{m}$.

Suppose now that for $T_{s}$ with $s<k, k>1$, (21)-(24) hold. Then for $k_{1}+\ldots+k_{m}=k-1$ we have

$$
\operatorname{supp} T_{k_{1}} * \ldots * T_{k_{m}} \subset \sum_{r=1}^{m} \operatorname{supp} T_{k_{r}} \subset \sum_{r=1}^{m} m_{k_{r}} Z=m_{k} Z,
$$

because $m_{k_{1}}+\ldots+m_{k_{m}}=m_{k}$. Here $\sum$ is the algebraic sum of sets.

By the properties of convolution, assumption (iii) and by (15) we obtain

$$
\begin{aligned}
\int_{Z^{m_{k}}}\left|H_{k}\left(z_{1}, \ldots, z_{m_{k}}\right)\right| e^{a\left(z_{1}+\ldots+z_{m_{k}}\right)} d z_{1} \ldots d z_{m_{k}} \\
=\sum_{r=1}^{\tilde{m}}\left(\begin{array}{c}
m \\
r
\end{array}\right) \sum_{\substack{k_{1}+\ldots+k_{r}=k-1 \\
k_{i} \geq 1}} \int_{Z^{k_{r}^{\prime}}} \int_{Z^{m-r}} \\
\quad \times \frac{\prod_{j=1}^{r}\left|H_{k_{j}}\left(u_{k_{j}}\right)\right| e^{a s\left(u_{k_{j}}\right)} \Phi\left(z_{k_{r}^{\prime}+1}\right) e^{a z_{k_{r}^{\prime}+1}} \ldots \Phi\left(z_{m_{k}}\right) e^{a z_{m_{k}}}}{\left|P\left(\sum_{j=1}^{r} s\left(u_{k_{j}}\right)+z_{k_{r}^{\prime}+1}+\ldots+z_{m_{k}}\right)\right|} d u d z \\
\leq \sum_{r=1}^{\tilde{m}}\left(\begin{array}{c}
m \\
r
\end{array}\right)_{\substack{k_{1}+\ldots+k_{r}=k-1 \\
k_{i} \geq 1}} B_{k_{1}}\left(C M_{\eta}\right)^{m_{k_{1}}} \ldots B_{k_{r}}\left(C M_{\eta}\right)^{m_{k_{r}}}\left(C M_{\eta}\right)^{m-r} \\
=B_{k}\left(C M_{\eta}\right)^{m_{k}},
\end{aligned}
$$

and

$$
\begin{aligned}
T_{k}[\phi]= & \alpha \sum_{r=1}^{\widetilde{m}}\left(\begin{array}{c}
m \\
r
\end{array}\right) \sum_{\substack{k_{1}+\ldots+k_{r}=k-1 \\
k_{i} \geq 1}} \int_{Z^{m_{k_{1}}}} \ldots \int_{Z^{m_{k_{r}}}} \int_{Z^{m-r}} \\
& \phi\left(\sum_{j=1}^{r} s\left(u_{j}\right)+z_{k_{r}^{\prime}+1}+\ldots+z_{m_{k}}\right) \\
& \times \frac{\prod_{j=1}^{r} \alpha^{k_{j}} H_{k_{j}}\left(u_{j}\right) \Phi\left(z_{k_{r}^{\prime}+1}\right) \ldots \Phi\left(z_{m_{k}}\right)}{P\left(\sum_{j=1}^{r} s\left(u_{j}\right)+z_{k_{r}^{\prime}+1}+\ldots+z_{m_{k}}\right)} d u_{1} \ldots d u_{r} d z \\
= & \alpha^{k} \int_{Z^{m_{k}}} \phi(u) H_{k}(u) d u .
\end{aligned}
$$


Moreover

$$
\begin{aligned}
\left|T_{k}[\phi]\right| \leq & |\alpha|^{k} \sup _{u \in m_{k} Z}|\phi(u)| e^{-a u} \sum_{r=1}^{\widetilde{m}}\left(\begin{array}{c}
m \\
r
\end{array}\right) \sum_{\substack{k_{1}+\ldots+k_{r}=k-1 \\
k_{i} \geq 1}} \\
& \prod_{j=1}^{r} \int_{Z_{m_{k}}}\left|H_{k_{j}}\left(u_{j}\right)\right| e^{a s\left(u_{j}\right)} d u_{j}\left(\int_{Z} \Phi(z) e^{a z} d z\right)^{m-r} \\
\leq & |\alpha|^{k} \sup _{u \in m_{k} Z}|\phi(u)| e^{-a u} \\
& \times \sum_{r=1}^{\widetilde{m}}\left(\begin{array}{c}
m \\
r
\end{array}\right) \sum_{k_{1}+\ldots+k_{r}=k-1} B_{k_{1}} \ldots B_{k_{r}}\left(C M_{\eta}\right)^{m_{k_{1}}+\ldots+m_{k_{r}}+m-r} \\
= & |\alpha|^{k} \sup _{u \in m_{k} Z}|\phi(u)| e^{-a u} B_{k}\left(C M_{\eta}\right)^{m_{k}} .
\end{aligned}
$$

By Lemma 2 we have

Corollary 1. The distribution $T_{k}$ defined by (19) satisfies the estimate

$$
\left|T_{k}[\phi]\right| \leq|\alpha|^{k}((m-1) k) ! D^{m_{k}} \sup _{z \in m_{k} Z}\left|\phi(z) e^{-a z}\right|
$$

with constant $D=C M_{\eta}$ independent of $a$, for $\phi \in L_{a}, m_{k}=(m-1) k+1$, $-a-b>\eta$.

3. Gevrey class of solutions. Let $J=\left\{J_{k}\right\}_{k \in \mathbb{N}}$ be a sequence of real numbers, and $\omega \in \mathbb{R}^{n}$.

Definition 1. We define $G^{\omega}(J)$ as the set of formal series $\sum_{k=1}^{\infty} T_{k}\left[e^{-x z}\right]$ with Laplace distributions $T_{k}$ such that the series $\sum_{k=1}^{\infty} \frac{1}{J_{k}} T_{k}\left[e^{-x z}\right]$ converges locally uniformly for $x>-\omega$.

ThEOREM 1. Fix $b=\left(b_{1}, b_{2}\right) \in \mathbb{R}^{2}$ and $\eta>0$, and let $\omega=-\eta-b$. Let $M_{\eta}=\int_{Z} e^{-\eta x} d x$, and $\Phi$ be a function in $C^{\infty}\left(\mathbb{R}_{+}^{2}\right)$ satisfying (9) for some constant $C<|\alpha|^{-1 /(m-1)} M_{\eta}^{-1}$. Define Laplace distributions $T_{k}$ by (10) and (19) for $k=0,1, \ldots$, and set $u_{k}(x)=T_{k}\left[e^{-x z}\right]$ for $x>-\omega$. Set $J_{k}=((m-1) k)$ !. Then the series

$$
\sum_{k=0}^{\infty} u_{k}(x)=\sum_{k=0}^{\infty} T_{k}\left[e^{-x z}\right]
$$

is a formal solution of (2) and it is in the Gevrey class $G^{\omega}(J)$.

Proof. The series (25) solves (2) by the construction of $T_{k}$. It remains to prove that it is in the Gevrey class. Since $e^{-x z} \in L_{a}$ (as a function of $z$ ) for 
every $a \geq-x$, from Corollary 1 it follows that if $x \geq-a>-\omega$, then

$$
\left|u_{k}(x)\right|=\left|T_{k}\left[e^{-x z}\right]\right| \leq|\alpha|^{k}\left(C M_{\eta}\right)^{(m-1) k+1} J_{k} \sup _{z \in m_{k} Z}\left|e^{-(a+x) z}\right| .
$$

Hence for $x \geq-a$, and in consequence for $x>-\omega$, we have

$$
\left|u_{k}(x)\right| \leq C M_{\eta} J_{k}\left(|\alpha|\left(C M_{\eta}\right)^{m-1}\right)^{k} .
$$

Since $|\alpha|\left(C M_{\eta}\right)^{m-1}<1$ the series $\sum_{k=1}^{\infty} u_{k}(x) / J_{k}$ is uniformly convergent on the set $\{x>-\omega\}$.

Fix $\beta>0$, and denote by $D_{k}$ the set

$$
D_{k}=\left\{y \in \mathbb{R}_{+}^{2}: y z>\beta k^{2} \text { for all } z \in k Z\right\} .
$$

TheOrem 2. Assume that the sequence $\left\{D_{k}\right\}_{k}$ is decreasing, and fix $\omega \in$ $\mathbb{R}^{2}$. Then, for the formal solution $\sum_{k=1}^{\infty} u_{k}(x)$ defined by $(25)$, the sequence

$$
\beta_{N}=\sup _{x \in D_{m_{N}}-\omega} \sum_{k=1}^{N}\left|u_{k}(x)\right|
$$

with $m_{N}=(m-1) N+1$, for $N \in \mathbb{N}$, is bounded.

Proof. We first observe that if $\left\{D_{k}\right\}$ decreases then so does $\left\{D_{k}-\omega\right\}$. If $x \in D_{m_{N}}-\omega$ and $r \leq N$ then $x \in D_{m_{N}}-a$ for some $a$ with $x \geq-a>-\omega$, so $x \in D_{m_{r}}-a$ and $(x+a) z \geq \beta m_{r}^{2}$ for $z \in m_{r} Z$. From the proof of Theorem 1 it follows that for such $x$ and $k=1,2, \ldots$,

$$
\left|u_{k}(x)\right| \leq C M_{\eta}((m-1) k) !\left(|\alpha|\left(C M_{\eta}\right)^{m-1}\right)^{k} \sup _{z \in m_{k} Z}\left|e^{-(a+x) z}\right| .
$$

Thus for all $r \leq N$,

$$
\left|u_{r}(x)\right| \leq C M_{\eta}((m-1) r) !\left(|\alpha|^{1 /(m-1)} C M_{\eta}\right)^{(m-1) r} e^{-\beta m_{r}^{2}}
$$

and

$$
\sum_{r=1}^{\infty}((m-1) r) ! E^{(m-1) r} e^{-\beta m_{r}^{2}} \leq \sum_{p=1}^{\infty} p ! E^{p} e^{-\beta(p+1)^{2}}<\infty
$$

with $E=|\alpha|^{1 /(m-1)} C M_{\eta}$.

It is worth pointing out that the solution (25) depends strictly on the choice of the function $\Phi$ in the formula (10). But it means in fact the dependence on the choice of the constant $b$ or $\omega=-b-\eta, \eta$ fixed.

We can now formulate some consequence of the possibility of this choice.

TheOREM 3. Fix $\eta>0$. For every $r, N \in \mathbb{N}$ we can choose $\omega>0$ and $a$ formal solution $\sum_{k=0}^{\infty} u_{k}(x)$ of $(2)$ such that

$$
\sum_{k=0}^{N}\left|u_{k}(x)\right| \leq C M_{\eta} \sum_{k=1}^{N}((m-1) k) ! E^{(m-1) k} e^{-\beta m_{k}^{2}}
$$

for $x \in D_{r}$ and $E=|\alpha|^{1 /(m-1)} C M_{\eta}$. 
Proof. If $r \geq m_{N}$, then obviously $D_{r} \subset D_{m_{N}} \subset D_{m_{N}}-\omega$ for every $\omega>0$, and (28) follows from Theorem 2. Suppose now that $r<m_{N}$. Then $m_{N} Z \subset r Z$, therefore, for $x \in D_{r}, x z>\beta r^{2}$ for all $z \in m_{N} Z$. If we choose $\omega \in D_{m_{N}}$ then $(x+\omega) z=x z+\omega z>\beta r^{2}+\beta m_{N}^{2}>\beta m_{N}^{2}$ for all $z \in m_{N} Z$, which means that $x \in D_{m_{N}}-\omega$, and the solution (25) defined for such $\omega$ satisfies (28).

4. Special case. Suppose now that the set $Z$ can be described by

$$
Z=\left\{(t, s) \in \mathbb{R}_{+}^{2}: s=f(t)\right\}=\left\{z(t): t \in \mathbb{R}_{+}\right\},
$$

where $z(t)=(t, f(t)), f: \mathbb{R}_{+} \rightarrow \mathbb{R}_{+}, f \in C^{\infty}, f^{\prime}<0, f^{\prime \prime}>0, f^{-1} \in C^{\infty}$ and

$$
\lim _{x \rightarrow 0} f(x)=\infty, \quad \lim _{x \rightarrow \infty} f(x)=0 .
$$

It is evident that the distribution $T_{0}$ defined by (10) with some constants $\eta>0, b=\left(b_{1}, b_{2}\right) \in \mathbb{R}_{+}^{2}$ fixed and a function $\Phi$ chosen to satisfy (9), can be written as an integral over $\mathbb{R}_{+}$:

$$
T_{0}[\phi]=\int_{0}^{\infty} \phi(t, f(t)) \Psi(t) d t
$$

for $\phi \in L_{(\omega)}, \omega=-\eta-b$, with $\Psi(t)=\Phi(t, f(t)) \sqrt{1+\left(f^{\prime}(t)\right)^{2}}$. It can be seen that for some constant $C^{\prime}<|\alpha|^{-1 /(m-1)} M_{\eta}^{-1}$ we have

$$
0<\Psi(t) \leq C^{\prime} e^{b z(t)}=C^{\prime} e^{b_{1} t+b_{2} f(t)} .
$$

Lemma 4. For every $k \in \mathbb{N}, k \geq 2$,

$$
k Z=\left\{(x, y) \in \mathbb{R}_{+}^{2}: y \geq k f(x / k)\right\}
$$

and $k Z$ is convex.

Proof. If $(x, y) \in k Z$, then there exist $t_{1}, \ldots, t_{k}>0$ such that $x=$ $t_{1}+\ldots+t_{k}$ and $y=f\left(t_{1}\right)+\ldots+f\left(t_{k}\right)$. Hence

$$
\frac{y}{k}=\frac{1}{k} f\left(t_{1}\right)+\ldots+\frac{1}{k} f\left(t_{k}\right) \geq f\left(\frac{t_{1}+\ldots+t_{k}}{k}\right)=f\left(\frac{x}{k}\right) .
$$

Now fix $(x, y) \in\{y \geq k f(x / k)\}$. Writing $t_{j}=x / k$ for $j=3, \ldots, k$, and $t_{1}=\alpha x / k, t_{2}=(2-\alpha) x / k$, for $\alpha \in(0,2)$ we have $x=t_{1}+\ldots+t_{k}$. Let $F(\alpha)=f(\alpha x / k)+f((2-\alpha) x / k)$. We see that $F(1)=2 f(x / k), F(\alpha) \rightarrow \infty$ as $\alpha \rightarrow 0$ or $\alpha \rightarrow 2$. Clearly $y-(k-2) f(x / k) \geq 2 f(x / k)$. Then by continuity of $F$, there exists $\alpha$ such that $F(\alpha)=y-(k-2) f(x / k)$. This proves that $y=f\left(t_{1}\right)+\ldots+f\left(t_{k}\right)$, so $(x, y) \in k Z$.

Let now $(x, y),(u, v) \in k Z$, and define $(r, s)=\lambda(x, y)+(1-\lambda)(u, v)$ for $0 \leq \lambda \leq 1$. We shall show that also $(r, s) \in k Z$. We have $r=\lambda x+(1-\lambda) u$, $s=\lambda y+(1-\lambda) v, y \geq k f(x / k)$ and $v \geq k f(u / k)$. Therefore 
$\frac{s}{k}=\lambda \frac{y}{k}+(1-\lambda) \frac{v}{k} \geq \lambda f\left(\frac{x}{k}\right)+(1-\lambda) f\left(\frac{u}{k}\right) \geq f\left(\frac{\lambda x+(1-\lambda) u}{k}\right)=f\left(\frac{r}{k}\right)$.

This proves the convexity of $k Z$.

Let $\sigma \in\{1,-1\}, V_{\sigma}=\left\{\left(t_{1}, t_{2}\right): t_{1}>0, t_{2}>0, \sigma\left(t_{2}-t_{1}\right)>0\right\}$ and consider the transformation $\Psi_{\sigma}: V_{\sigma} \rightarrow 2 Z$ given by

$$
\Psi_{\sigma}\left(t_{1}, t_{2}\right)=\left(t_{1}+t_{2}, f\left(t_{1}\right)+f\left(t_{2}\right)\right)
$$

for $t=\left(t_{1}, t_{2}\right) \in V_{\sigma}$. The Jacobian $J \Psi_{\sigma}(t)=\left|f^{\prime}\left(t_{2}\right)-f^{\prime}\left(t_{1}\right)\right| \neq 0$ on $V_{\sigma}$, hence $\Psi_{\sigma}$ is invertible and the Jacobian $J \Psi_{\sigma}^{-1}(u)$ is independent of $\sigma$. We use the notation $\Psi_{+}^{-1}(u)=\left(t_{1}(u), t_{2}(u)\right)$ and $\Psi_{-}^{-1}(u)=\left(t_{2}(u), t_{1}(u)\right)$, and

$$
K(u)= \begin{cases}J \Psi_{\sigma}^{-1}(u) & \text { for } u \in 2 Z \\ 0 & \text { for } u \in \mathbb{R}_{+}^{2} \backslash 2 Z .\end{cases}
$$

Define $\widehat{Z}=\left\{(x, y) \in \mathbb{R}_{+}^{2}: y>f(x)\right\}$, and for $v \in \widehat{Z}$ denote by $T_{v}$ the open interval $\left(f^{-1}\left(v_{2}\right), v_{1}\right)$. It follows from the assumptions on $Z$ that if $t \in T_{v}$ then $v_{1}-t>0$ and $v_{2}-f(t)>0$.

Define

$$
\widehat{K}(v)= \begin{cases}\int_{T_{v}} K(v-z(t)) d t & \text { for } v \in \widehat{Z}, \\ 0 & \text { for } v \in \mathbb{R}_{+}^{2} \backslash \widehat{Z} .\end{cases}
$$

LEMma 5. For every $k \in \mathbb{N}, k \geq 2$ and for $\phi \in L_{(\omega)}$ we have

$$
T_{0}^{* k}[\phi]=\int_{\mathbb{R}_{+}^{2}} \phi(z) \Phi_{k}(z) d z
$$

where

$$
\begin{aligned}
& \Phi_{2}(z):= \begin{cases}2 \Psi\left(t_{1}(z)\right) \Psi\left(t_{2}(z)\right) K(z) & \text { for } z \in 2 Z, \\
0 & \text { for } z \in \mathbb{R}_{+}^{2} \backslash 2 Z,\end{cases} \\
& \Phi_{k}(v):= \begin{cases}\int_{T_{v}} \Phi_{k-1}(v-z(t)) \Psi(t) d t & \text { for } v \in \widehat{Z}, \\
0 & \text { for } v \in \mathbb{R}_{+}^{2} \backslash \widehat{Z},\end{cases}
\end{aligned}
$$

for $k \geq 3$. Moreover, $\Phi_{k} \in C^{\infty}\left((k Z)^{\circ}\right), \operatorname{supp} \Phi_{k} \subset k Z$ and

$$
\begin{aligned}
& \Phi_{k} \leq \Theta_{k}:= \begin{cases}\Phi_{2}^{* p} & \text { for } k=2 p, \\
\Phi_{3} * \Phi_{2}^{* p-1} & \text { for } k=2 p+1,\end{cases} \\
& \Phi_{3} * \Phi_{3} \leq \Phi_{2}^{* 3} .
\end{aligned}
$$

Proof. By definition of convolution of distributions and by formula (29) we can see that

$$
T_{0}^{* 2}[\phi]=\int_{0}^{\infty} \int_{0}^{\infty} \phi(z(t)+z(s)) \Psi(t) \Psi(s) d s d t
$$


Now we use the elementary fact that

$$
\int_{0}^{\infty} \int_{0}^{\infty}=\int_{0}^{\infty} \int_{0}^{t}+\int_{0}^{\infty} \int_{t}^{\infty}
$$

and we change variables using transformations (32). Thus

$$
T_{0}^{* 2}[\phi]=2 \int_{2 Z} \phi(u) \Psi\left(t_{1}(u)\right) \Psi\left(t_{2}(u)\right) J \Psi_{\sigma}^{-1}(u) d u=\int_{\mathbb{R}_{+}^{2}} \phi(u) \Phi_{2}(u) d u .
$$

Obviously supp $\Phi_{2} \subset 2 Z$ and regularity of $\Phi_{2}$ follows from regularity of $\Psi$ and $\Psi_{\sigma}^{-1}$.

Similarly, since $T_{0}^{* 3}=T_{0}^{* 2} * T_{0}$, we get

$$
T_{0}^{* 3}[\phi]=\int_{\mathbb{R}_{+}^{2}} \int_{0}^{\infty} \phi(u+z(t)) \Phi_{2}(u) \Psi(t) d u d t
$$

The mapping

$$
\chi\left(u_{1}, u_{2}, t\right)=\left(u_{1}+t, u_{2}+f(t), t\right)
$$

is a homeomorphism

$$
\chi:(0, \infty)^{3} \rightarrow\left\{\left(v_{1}, v_{2}, \tau\right): v_{2}>f\left(v_{1}\right), \tau \in T_{v}\right\}
$$

with Jacobian 1. Indeed, if $v_{1}=u_{1}+t, v_{2}=u_{2}+f(t)$ for $u_{1}>0, u_{2}>0$, $t>0$, then

$$
v_{2}=u_{2}+f\left(v_{1}-u_{1}\right)>f\left(v_{1}-u_{1}\right)>f\left(v_{1}\right),
$$

because $f$ decreases. Similarly, $t=v_{1}-u_{1}<v_{1}$, and since $f(t)=v_{2}-u_{2}$ we have $t=f^{-1}\left(v_{2}-u_{2}\right)>f^{-1}\left(v_{2}\right)$. Thus $t \in T_{v}$. Suppose now that $v_{2}>f\left(v_{1}\right)$ and $t \in T_{v}$. Then $u_{1}:=v_{1}-t>0$ and $u_{2}:=v_{2}-f(t)>0$, so $\left(u_{1}, u_{2}, t\right)$ belongs to the domain of $\chi$. The injectivity and continuity of $\chi$ are obvious. Thus we can use $\chi$ to change variables in the last integral to obtain

$$
T_{0}^{* 3}[\phi]=\int_{\widehat{Z}} \phi(v) \int_{T_{v}} \Phi_{2}(v-z(t)) \Psi(t) d t d v=\int_{\mathbb{R}_{+}^{2}} \phi(v) \Phi_{3}(v) d v
$$

where $\Phi_{3}$ is given by (35) for $k=3$.

If $v \in\left(\mathbb{R}_{+}^{2} \backslash(k+1) Z\right) \cap \widehat{Z}$ then $v-z(t) \in \mathbb{R}_{+}^{2} \backslash k Z$ for $t \in T_{v}$, so $\operatorname{supp} \Phi_{3} \subset 3 Z$ and obviously $\Phi_{3} \in C^{\infty}\left((3 Z)^{\circ}\right)$. We now proceed by induction.

Since $T_{0}^{* k+1}=T_{0}^{* k} * T_{0}$, we have

$$
\begin{aligned}
T_{0}^{* k+1}[\phi] & =\int_{\mathbb{R}_{+}^{2}} \int_{0}^{\infty} \phi(u+z(t)) \Phi_{k}(u) \Psi(t) d t d u \\
& =\int_{\widehat{Z}} \phi(v) \int_{T_{v}} \Phi_{k}(v-z(t)) \Psi(t) d t d v=\int_{\mathbb{R}_{+}^{2}} \phi(v) \Phi_{k+1}(v) d v
\end{aligned}
$$


From (35) it follows that

$$
\begin{aligned}
\Phi_{k+1}(v) & =\int_{T_{v}} \int_{T_{v-z(t)}} \Phi_{k-1}(v-z(t)-z(s)) \Psi(t) \Psi(s) d t d s \\
& =\int_{\Psi_{\sigma}\left(\widehat{T}_{v}\right)} \Phi_{k-1}(v-y) \Phi_{2}(y) d y
\end{aligned}
$$

where $\widehat{T}_{v}=\left\{(t, s) \in \mathbb{R}_{+}^{2}: t \in T_{v}, s<t\right\}$.

The estimate (36) is obvious for $k=2$ and $k=3$. Suppose that it is true for $k=2 p$ and $k=2 p+1$. Then

$$
\begin{aligned}
\Phi_{2 p+2}(v) & =\int_{\Psi_{\sigma}\left(\widehat{T}_{v}\right)} \Phi_{2 p}(v-y) \Phi_{2}(y) d y \\
& \leq \int_{\Psi_{\sigma}\left(\widehat{T}_{v}\right)} \Phi_{2}^{* p}(v-y) \Phi_{2}(y) d y \leq \Phi_{2}^{*(p+1)}(v),
\end{aligned}
$$

and

$$
\begin{aligned}
\Phi_{2 p+3}(v) & =\int_{\Psi_{\sigma}\left(\widehat{T}_{v}\right)} \Phi_{2 p+1}(v-y) \Phi_{2}(y) d y \\
& \leq \int_{\Psi_{\sigma}\left(\widehat{T}_{v}\right)} \Phi_{3} * \Phi_{2}^{*(p-1)}(v-y) \Phi_{2}(y) d y \leq \Phi_{3} * \Phi_{2}^{* p}(v) .
\end{aligned}
$$

By (35), using the transformations $\chi$ and $\Psi_{\sigma}$ we get

$$
\begin{aligned}
\Phi_{3} * \Phi_{3}(v) & =\int_{0}^{v} \Phi_{3}(v-u) \Phi_{3}(u) d u \\
& =\int_{0}^{v} \int_{T_{v-u}} \int_{T_{u}} \Phi_{2}(v-u-z(t)) \Phi_{2}(u-z(s)) \Psi(t) \Psi(s) d t d s d u \\
& =\int_{0}^{v} \int_{T_{y}} \int_{T_{y-z(t)}} \Phi_{2}(v-y) \Phi_{2}(y-(z(t)+z(s))) \Psi(t) \Psi(s) d t d s d y \\
& \leq \int_{0}^{v} \int_{\Psi_{\sigma}\left(\widehat{T}_{y}\right)} \Phi_{2}(v-y) \Phi_{2}(y-z) \Phi_{2}(z) d z d y \leq \Phi_{2}^{* 3}(v) .
\end{aligned}
$$

Corollary 2. The function $\Theta_{k}$ defined by (36) for $k=2,3, \ldots$ satisfies, for $v \in \mathbb{R}_{+}^{2}$,

$$
\Theta_{k}(v) \leq\left(\sqrt{2} C^{\prime}\right)^{k} e^{b v} \mathcal{K}_{k}(v)
$$

where

$$
\mathcal{K}_{n}(u)= \begin{cases}K^{* p}(u) & \text { for } n=2 p \\ \widehat{K} * K^{* p-1}(u) & \text { for } n=2 p+1\end{cases}
$$


Proof. By the definition of $\Phi_{2}$ it follows immediately that

$$
\Phi_{2}(v) \leq 2 C^{\prime 2} e^{b\left(z\left(t_{1}(v)\right)+z\left(t_{2}(v)\right)\right)} K(v)=\left(\sqrt{2} C^{\prime}\right)^{2} e^{b v} K(v),
$$

since $z\left(t_{1}(v)\right)+z\left(t_{2}(v)\right)=v$. Similarly,

$$
\begin{aligned}
\Phi_{3}(v) & \leq 2 C^{\prime 3} \int_{T_{v}} e^{b(v-z(t))} K(v-z(t)) e^{b z(t)} d t \\
& =2 C^{\prime 3} e^{b v} \int_{T_{v}} K(v-z(t)) d t<\left(\sqrt{2} C^{\prime}\right)^{3} e^{b v} \widehat{K}(v) .
\end{aligned}
$$

Hence, for $k=2 p$ we have

$$
\Theta_{k}(v)=\Phi_{2}^{* p}(v) \leq\left(\sqrt{2} C^{\prime}\right)^{2 p} e^{b v} K^{* p}(v)
$$

and for $k=2 p+1$,

$$
\Theta_{k}(v)=\Phi_{3} * \Phi_{2}^{* p-1}(v) \leq\left(\sqrt{2} C^{\prime}\right)^{2 p+1} e^{b v} \widehat{K} * K^{* p-1}(v) .
$$

Exactly as in the general case, for $T_{0}$ defined by (29), we can define $T_{k}$ by (19).

TheOREM 4. The distribution $T_{k}$ defined by (19) acts on test functions as a function $G_{k} \in C^{\infty}\left(\left(m_{k} Z\right)^{\circ}\right)\left(m_{k}=(m-1) k+1\right)$ defined on $\mathbb{R}_{+}^{2}$ by the recurrence formula

$$
\begin{aligned}
& G_{1}(u)=\alpha \frac{\Phi_{m}(u)}{P(u)} \\
& G_{k}(u)=\frac{\alpha}{P(u)} \sum_{r=1}^{\tilde{m}}\left(\begin{array}{c}
m \\
r
\end{array}\right) \sum_{\substack{k_{1}+\ldots+k_{r}=k-1 \\
k_{i} \geq 1}} G_{k_{1}} * \ldots * G_{k_{r}} * \Phi_{m-r}(u)
\end{aligned}
$$

for $k>1$ and $\widetilde{m}=\min \{m, k-1\}$. Moreover, we have the estimate

$$
\left|G_{k}(u)\right| \leq|\alpha|^{k}((m-1) k) ! e^{b u}\left(\sqrt{2} C^{\prime}\right)^{m_{k}} \mathcal{K}_{m_{k}}(u) .
$$

Proof. The functions $G_{k}(k=1,2, \ldots)$ are well defined because $\Phi_{m}$ and $G_{k_{1}} * \ldots * G_{k_{r}} * \Phi_{m-r}$ vanish on some neighbourhoods of $Z$. By (19),

and

$$
T_{1}=\frac{\alpha}{P} T_{0}^{* m}
$$

$$
T_{k}=\frac{\alpha}{P} \sum_{r=1}^{\tilde{m}}\left(\begin{array}{c}
m \\
r
\end{array}\right) \sum_{\substack{k_{1}+\ldots+k_{r}=k-1 \\
k_{i} \geq 1}} T_{k_{1}} * \ldots * T_{k_{r}} * T_{0}^{* m-r} .
$$

Then for every test function $\phi$ we have

$$
T_{1}[\phi]=\alpha \int_{\mathbb{R}_{+}^{2}} \phi(u) \frac{\Phi_{m}(u)}{P(u)} d u=\int_{\mathbb{R}_{+}^{2}} \phi(u) G_{1}(u) d u,
$$


and if for $s<k, T_{s}[\phi]=\int_{\mathbb{R}_{+}^{2}} \phi(u) G_{s}(u) d u$, then for $u_{1}, \ldots, u_{r}, v \in \mathbb{R}_{+}^{2}$,

$T_{k}[\phi]$

$=\alpha \sum_{r=1}^{\widetilde{m}}\left(\begin{array}{c}m \\ r\end{array}\right) \sum_{\substack{k_{1}+\ldots+k_{r}=k-1 \\ k_{i} \geq 1}} \int_{\substack{\left(\mathbb{R}_{+}^{2}\right)^{r+1} \\ P\left(\sum_{j=1}^{r} u_{j}+v\right)}} \frac{\phi\left(\sum_{j=1}^{r} u_{j}+v\right)}{P} G_{k_{j}}\left(u_{j}\right) \Phi_{m-r}(v) d u d v$

$=\alpha \sum_{r=1}^{\tilde{m}}\left(\begin{array}{c}m \\ r\end{array}\right) \sum_{\substack{k_{1}+\ldots+k_{r}=k-1 \\ k_{i} \geq 1}} \int_{\mathbb{R}_{+}^{2}} \phi(z) \frac{G_{k_{1}} * \ldots * G_{k_{r}} * \Phi_{m-r}(z)}{P(z)} d z$.

Hence we get formula (40) for $G_{k}$ with $k>1$. It remains to prove the estimate (41). By Corollary 2 it is sufficient to show that

$$
\left|G_{k}(u)\right| \leq|\alpha|^{k}((m-1) k) ! \Theta_{m_{k}}(u),
$$

and for this purpose we proceed by induction. Of course, for $k=1$, (42) holds. Assume it holds for $s<k$. Then for $k_{1}+\ldots+k_{r}=k-1$ we have

$$
\begin{aligned}
& \left|G_{k_{1}} * \ldots * G_{k_{r}} * \Phi_{m-r}(u)\right| \\
& \quad \leq|\alpha|^{k_{1}+\ldots+k_{r}}\left((m-1) k_{1}\right) ! \ldots\left((m-1) k_{r}\right) ! \Theta_{m_{k_{1}}} * \ldots * \Theta_{m_{k_{r}}} * \Theta_{m-r}(u) \\
& \quad \leq|\alpha|^{k-1}((m-1)(k-1)) ! \Theta_{m_{k}}(u) .
\end{aligned}
$$

The rest of the reasoning runs as in the proof of Lemma 2.

Now we can state an analogue of Theorem 1.

ThEOREM 5. Fix $b=\left(b_{1}, b_{2}\right) \in \mathbb{R}^{2}$ and $\eta>0$ and set $\omega=-\eta-b$. Assume that, for some constant $A_{\eta}>1$,

$$
\int_{\mathbb{R}_{+}^{2}} e^{-\eta z} K(z) d z \leq A_{\eta}, \quad \int_{\mathbb{R}_{+}^{2}} e^{-\eta z} \widehat{K}(z) d z \leq A_{\eta},
$$

where $K$ and $\widehat{K}$ are defined by (33) and (34) respectively. Let $\Psi \in C^{\infty}\left(\mathbb{R}_{+}^{2}\right)$ satisfy (30) for some constant $C^{\prime}<|\alpha|^{-1 /(m-1)}\left(2 A_{\eta}\right)^{-1 / 2}, G_{k}$ be given by (39) and (40) for $k=1,2, \ldots$, and let

$$
\begin{aligned}
& u_{0}(x)=\int_{0}^{\infty} e^{-x z(t)} \Psi(t) d t, \\
& u_{k}(x)=\int_{\mathbb{R}_{+}^{2}} e^{-x z} G_{k}(z) d z
\end{aligned}
$$

for $k \geq 1$ and $x>-\omega$. Set $J_{k}=((m-1) k)$ ! and $J=\left\{J_{k}\right\}$. Then the series $\sum_{k=0}^{\infty} u_{k}(x)$ is a formal solution of (2) and is in the Gevrey class $G^{\omega}(J)$.

Proof. We note that the function $K$ depends on the curve $Z$ only, therefore the constant $A_{\eta}$ depends on $Z$ and $\eta$ only. By assumption, definition of 
$\mathcal{K}_{k}$ and properties of convolution we have, for $k=2 p$,

$$
\int_{\mathbb{R}_{+}^{2}} e^{-\eta z} \mathcal{K}_{k}(z) d z=\int_{\mathbb{R}_{+}^{2}} e^{-\eta z} K^{* p}(z) d z \leq\left(\int_{\mathbb{R}_{+}^{2}} e^{-\eta z} K(z) d z\right)^{p} \leq A_{\eta}^{p}=A_{\eta}^{k / 2},
$$

and for $k=2 p+1$,

$$
\begin{aligned}
\int_{\mathbb{R}_{+}^{2}} e^{-\eta z} \mathcal{K}_{k}(z) d z & =\int_{\mathbb{R}_{+}^{2}} e^{-\eta z} \widehat{K} * K^{* p-1}(z) d z \\
& \leq \int_{\mathbb{R}_{+}^{2}} e^{-\eta z} \widehat{K}(z) d z\left(\int_{\mathbb{R}_{+}^{2}} e^{-\eta z} K(z) d z\right)^{p-1} \leq A_{\eta}^{p}<A_{\eta}^{k / 2}
\end{aligned}
$$

From Theorem 4 we obtain, for $x>-\omega$,

$$
\int_{\mathbb{R}_{+}^{2}} e^{-x z} G_{k}(z) d z \leq|\alpha|^{k}((m-1) k) !\left(\sqrt{2} C^{\prime}\right)^{m_{k}} \int_{\mathbb{R}_{+}^{2}} e^{-(x-b) z} \mathcal{K}_{m_{k}}(z) d z .
$$

We conclude from the definition of $\omega$ that for $x>-\omega$ we have $x-b>$ $-\omega-b=\eta$, hence

$$
\begin{aligned}
\int_{\mathbb{R}_{+}^{2}} e^{-x z} G_{k}(z) d z & \leq|\alpha|^{k}((m-1) k) !\left(\sqrt{2 A_{\eta}} C^{\prime}\right)^{(m-1) k+1} \\
& =\sqrt{2 A_{\eta}} C^{\prime}((m-1) k) !\left(|\alpha|\left(\sqrt{2 A_{\eta}} C^{\prime}\right)^{m-1}\right)^{k} .
\end{aligned}
$$

By the assumption on $C^{\prime}$, we have $F_{\eta}:=|\alpha|\left(\sqrt{2 A_{\eta}} C^{\prime}\right)^{m-1}<1$, and

$$
\frac{u_{k}(x)}{((m-1) k) !} \leq \sqrt{2 A_{\eta}} C^{\prime} F_{\eta}^{k}
$$

therefore the theorem is proved.

Similarly, we can rephrase Theorem 2 as follows.

Theorem 6. Suppose the sequence $\left\{D_{k}\right\}_{k}$ decreases and let $\omega=-\eta-b \in$ $\mathbb{R}^{2}$ be as in Theorem 5. Then for the formal solution (43) and (44) the sequence (27) with $m_{N}=(m-1) N+1$, for $N \in \mathbb{N}$, is bounded.

Proof. If $x \in D_{m_{k}}-\omega$ then we have $(x-b) z>\eta z+\beta\left(m_{k}\right)^{2}$. Hence

$$
\begin{aligned}
u_{k}(x) & =\int_{\mathbb{R}_{+}^{2}} e^{-x z} G_{k}(z) d z \leq|\alpha|^{k}\left(\sqrt{2} C^{\prime}\right)^{m_{k}}((m-1) k) ! \int_{\mathbb{R}_{+}^{2}} e^{-(x-b) z} \mathcal{K}_{m_{k}}(z) d z \\
& \leq \sqrt{2 A_{\eta}} C^{\prime} F_{\eta}^{k}((m-1) k) ! e^{-\beta m_{k}^{2}}
\end{aligned}
$$

and the rest of the proof runs as in the general case.

5. Example. In this section we show that the assumptions in the present work are not vacuous, that is, there exists a polynomial $P$ which has the asserted properties. Suppose that

$$
P(z)=P\left(z_{1}, z_{2}\right)=Q(z)\left(z_{1} z_{2}-1\right),
$$


where $Q$ is some polynomial with $|Q|>1$ on $\mathbb{R}_{+}^{2}$. Then

$$
\text { Char } P \cap \mathbb{R}_{+}^{2}=\left\{z: z_{2}=1 / z_{1}\right\}
$$

and we can choose the curve $Z=\left\{(t, 1 / t): t \in \mathbb{R}_{+}\right\} \subset$ Char $P \cap \mathbb{R}_{+}^{2}$. It is easy to check that $k Z=\left\{x \in \mathbb{R}_{+}^{2}: x_{2} \geq k^{2} / x_{1}\right\}$, therefore conditions (i)-(iii) hold.

To check (iv), we observe that for $c_{i} \geq \eta>0$,

$$
\begin{aligned}
\int_{Z} e^{-c z} d z & =\int_{0}^{\infty} e^{-c_{1} t-c_{2} 1 / t} \sqrt{1+1 / t^{4}} d t \leq \int_{0}^{1} e^{-c_{2} 1 / t} \sqrt{1+1 / t^{4}} d t+\sqrt{2} \int_{1}^{\infty} e^{-c_{1} t} d t \\
& =\int_{1}^{\infty} e^{-c_{2} s} \sqrt{1+1 / s^{4}} d s+\frac{\sqrt{2} e^{-c_{1}}}{c_{1}} \leq \sqrt{2}\left(\frac{e^{-c_{2}}}{c_{2}}+\frac{e^{-c_{1}}}{c_{1}}\right)<2 \sqrt{2} \frac{e^{-\eta}}{\eta} .
\end{aligned}
$$

Clearly, the function $f(t)=1 / t$ for $t \in \mathbb{R}_{+}$satisfies the conditions assumed at the beginning of Section 5 . We show that in this example the set $D_{k}$ defined by (26) can be written as

$$
D_{k}=\left\{y \in \mathbb{R}_{+}^{2}: y_{2}>\beta^{2} \frac{k^{2}}{4 y_{1}}\right\} .
$$

Indeed, fix $y=\left(y_{1}, y_{2}\right) \in D_{k}$. The function

$$
F(z)=F\left(z_{1}, z_{2}\right)=y z-\beta k^{2}=y_{1} z_{1}+y_{2} z_{2}-\beta k^{2}
$$

defined for $z \in k Z$ satisfies $F(z) \geq 0$ on $k Z$. We have $\frac{\partial F}{\partial z_{1}}(z)=y_{1}>0$, $\frac{\partial F}{\partial z_{2}}(z)=y_{2}>0$ for all $z \in k Z$, therefore $\min \{F(z): z \in k Z\} \geq 0$ must be attained on the boundary $\partial(k Z)=\left\{z_{2}=k^{2} / z_{1}\right\}$. To calculate this minimal value we consider the function $F$ on $\partial(k Z)$ as a function of one variable $z_{1}$, that is, as the function

$$
h\left(z_{1}\right)=F\left(z_{1}, \frac{k^{2}}{z_{1}}\right)=y_{1} z_{1}+y_{2} \frac{k^{2}}{z_{1}}-\beta k^{2} .
$$

Since $h^{\prime}\left(z_{1}\right)=y_{1}-y_{2} k^{2} / z_{1}^{2}=0$ for $z_{1}=k \sqrt{y_{2} / y_{1}}$, we have

$$
\begin{aligned}
\min \{F(z): z \in k Z\} & =\min \{F(z): z \in \partial(k Z)\}=h\left(k \sqrt{y_{2} / y_{1}}\right) \\
& =k\left(2 \sqrt{y_{1} y_{2}}-\beta k\right) \geq 0
\end{aligned}
$$

for $y_{1} y_{2} \geq(\beta k)^{2} / 4$.

Conversely, if $y$ satisfies the last inequality, then the quadratic function $g\left(z_{1}\right)=z_{1} h\left(z_{1}\right)=y_{1} z_{1}^{2}-\beta k^{2} z_{1}+y_{2} k^{2}$ has determinant $\Delta=\beta^{2} k^{4}-4 y_{1} y_{2} k^{2}=$ $k^{2}\left(\beta^{2} k^{2}-4 y_{1} y_{2}\right) \leq 0$, so $h\left(z_{1}\right)=g\left(z_{1}\right) / z_{1} \geq 0$ for all $z_{1}>0$. Hence, for all $z \in k Z, F(z) \geq h\left(z_{1}\right) \geq 0$ and consequently $y \in D_{k}$.

Thus the sequence $\left\{D_{k}\right\}_{k}$ decreases.

The transformation $\Psi_{\sigma}$ has the form

$$
t_{1}+t_{2}=u_{1}, \quad 1 / t_{1}+1 / t_{2}=u_{2},
$$


and the inverse transformation $\Psi_{+}^{-1}$ is given by the formula

$$
t_{1}(u)=\frac{1}{2}\left(u_{1}-\sqrt{u_{1}\left(u_{1}-4 / u_{2}\right)}\right), \quad t_{2}(u)=\frac{1}{2}\left(u_{1}+\sqrt{u_{1}\left(u_{1}-4 / u_{2}\right)}\right) .
$$

Hence, we get the Jacobian

$$
K(u)=\frac{u_{1}}{u_{2}^{2} \sqrt{u_{1}\left(u_{1}-4 / u_{2}\right)}}=\frac{1}{u_{2} \sqrt{u_{2}\left(u_{2}-4 / u_{1}\right)}} .
$$

Since $\int_{2 Z}=\int_{0}^{\infty} \int_{4 / u_{1}}^{\infty}$ and $\int_{4 / u_{1}}^{\infty} K\left(u_{1}, u_{2}\right) d u_{2}=u_{1} / 2$, for any $\eta>0$ we obtain

$$
\begin{array}{rl}
\int_{\mathbb{R}_{+}^{2}} e^{-\eta u} & K(u) d u=\int_{2 Z} e^{-\eta u} K(u) d u \\
& =\int_{0}^{\infty} e^{-\eta_{1} u_{1}}\left[\int_{4 / u_{1}}^{\infty} e^{-\eta_{2} u_{2}} K\left(u_{1}, u_{2}\right) d u_{2}\right] d u_{1} \leq \frac{1}{2} \int_{0}^{\infty} e^{-\eta_{1} u_{1}} u_{1} d u_{1}=\frac{1}{2 \eta_{1}^{2}} .
\end{array}
$$

Similar, but slightly more sophisticated calculations show that the function $\widehat{K}$ in this case also satisfies the assumptions of Theorem 5 .

\section{References}

[G-T] R. Gerard and H. Tahara, Singular Nonlinear Partial Differential Equations, Aspects of Mathematics, Vieweg, Wiesbaden, 1996.

[P-Z] M. E. Pliś and B. Ziemian, Convolution equations in the space of Laplace distributions, Ann. Polon. Math. 69 (1998), 271-281.

[S-Z] Z. Szmydt and B. Ziemian, Laplace distributions and hyperfunctions on $\overline{\mathbb{R}}_{+}^{n}$, J. Math. Sci. Univ. Tokyo 5 (1998), 41-74.

Pedagogical University

Podchorążych 2

30-084 Kraków, Poland

E-mail: meplis@wsp.krakow.pl 